\title{
A Sample of Folk Poetry of the Sui: The Volume of Ancestor Worship
}

DOI: http://dx.doi.org/10.12775/LC.2021.020

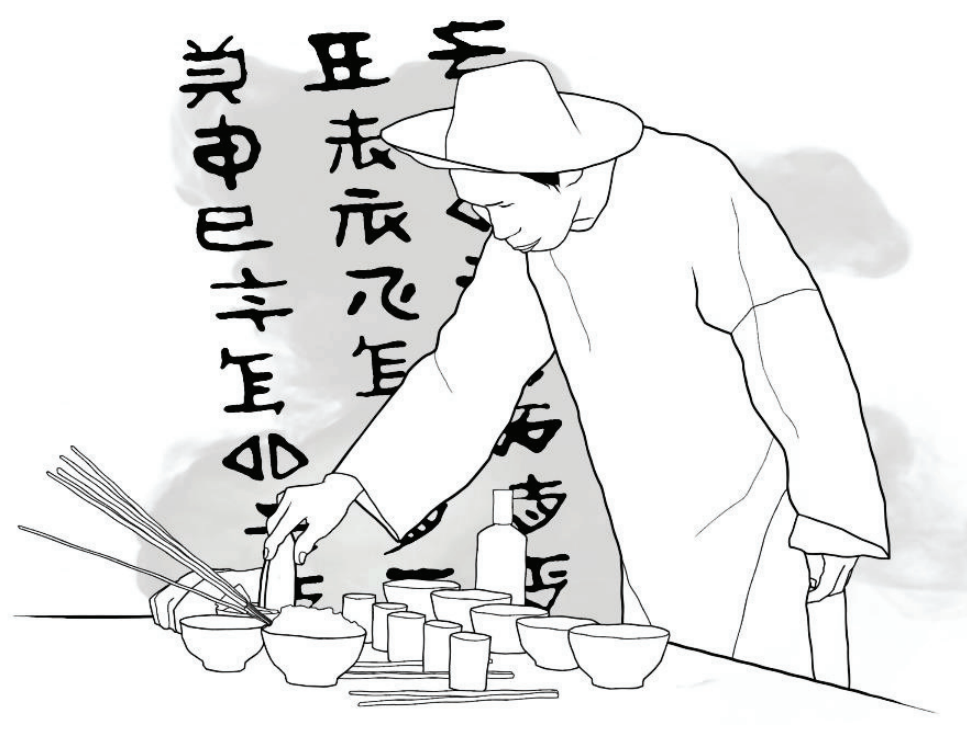

* PhD, an assistant professor at the Institute of Oriental Studies, Adam Mickiewicz University in Poznań. His research interests concern languages and cultures of ethnic minorities in China, especially the Sui people, as well as broadly understood sinological linguistics.

E-mail: kamil@burkiewicz.pl | ORCID: 0000-0002-7839-1051. 
The proper execution of a relevant ritual is, however, far beyond the knowledge accessible to ordinary people and requires the use of services provided by shamans known as [pju ${ }^{1}$ ta: $\left.i^{3}\right]$. The most capable are also referred to as $\left[\right.$ Pai $^{3}$ ha: $\left.: y^{6} \mathrm{le}^{1}\right]$, i.e. those who have mastered $\left[1 \mathrm{e}^{1}\right.$ sui $\left.^{3}\right]$ - the original writing system and the divination books written using it.

When invited to perform a ritual of ancestor worship, a shaman starts his preparations by setting up sacrificial goods and the necessary utensils. Right in front of the $\left[6 \mathrm{ir}^{3} \mathrm{qon}^{5}\right.$ $\mathrm{pu}^{4}$ ] - a special place in every family's home dedicated to male forebears - a long table is placed with one bench on both sides. Subsequently, the following items are arranged in a specific manner: tofu, boiled pork, a piglet, a bowl of salt and hot pepper, ten joss sticks, joss paper, five cups, five pairs of chopsticks, two pieces of bamboo called [bin ${ }^{5}$ ], a smoking pipe, a bowl of rice, another two bowls of boiled glutinous rice and, finally, some farm tools. After everything is put in the right order, the shaman picks up a pair of chopsticks in his left hand, and then uses the other hand to put some rice in his mouth. He doesn't swallow it, though, but spits the rice out in the direction of the $\left[\mathrm{Gi}^{3} \mathrm{qo \eta}^{5} \mathrm{pu}^{4}\right]$. Now, it is the time to start the recitation of The Volume of Ancestor Worship - the proper ritual thus begins.

The ritual depicted above is merely a small sample picked out of the great variety of traditional beliefs and customs still practised by the Sui people ${ }^{1}$ one of the ethnic minorities of China, officially recognised by the People's Government, and that inhabiting mainly the southern-eastern part of Guizhou Province, and particularly the Sandu Sui Autonomous County ${ }^{2}$. With a total population exceeding 400,000 people, the modern Sui successfully preserve their own language, classified within the Tai-Kadai family, as well as a logographic writing system, applied strictly for divination purposes ${ }^{3}$. The latter was the object of in-depth study presented in this author's $\mathrm{PhD}$ thesis (2015), which is supplemented with an additional introduction of the Sui ethnic group, its language and culture.

Among hundreds of volumes of mysterious books whose meaning and application were until relatively recently a secret strictly guarded by Sui shamans, there is one dedicated to the custom of $\left[\mathrm{he}^{4}\right.$ ha: $\mathrm{u}^{3} \mathrm{qo \eta}^{5} \mathrm{tsje}^{1}$ ], literally "preparing an alcohol offering for a male ancestor". In Chinese publications, the book is often referred to as The Volume of Ancestor Worship (Jizu Jing 祭祖经). As in the case of all other Sui written literary pieces, its textual layer conveys only a part of the whole content. Therefore, during ritual recitation, a shaman must rely on his memory to deliver all the passages that he learned from his master in an oral form. For this reason, the contents of volumes that bear the same title, but that have passed among different lineages of shamans, may differ considerably. The one presented here was recorded, notated phonetically and translated into Chinese by Wei Shuqi 韦述启, a native Sui researcher, during his visits to Wei Chaoxian 韦朝贤, a renowned “master of the books" who until his death in 2008 lived in the Sandong Township 4 .

\footnotetext{
1 Sui: [sui ${ }^{3}$ ], Chinese: Shui 水. This article employs IPA for all Sui language terms and names. Each syllable is annotated with superscripted numbers from one to eight, representing respective tones (cf. Burkiewicz 2015: 67). Chinese terminology appears both in a Romanised form according to the Hanyu Pinyin standard and as the original writing, represented by simplified Chinese characters.

2 Sui: [ha:m'pa'], Chinese: Sandou Shuizu Zizhixian 三都水族自治县.

3 The illustration on the initial page is intended to give an impression of the Sui script appearance. The characters used in the background come from The Volume of the First and Seventh Months, the Volume of the Ren and Chen (Wang 1994: 319).

${ }^{4}$ Sui: $\left[\operatorname{cian}^{5}\right]$, Chinese: Sandong Xiang 三洞乡. Sandong Township is located in the central part of Sandu County.
} 
The whole text of The Volume of Ancestor Worship can be found in Wei Shuqi's master's thesis (2012). It consists of nearly two thousand verses grouped into sixteen chapters, representing specific stages of the ritual. This paper, being only a brief glimpse into the subject, provides an English translation of the first 55 verses of the initial chapter, starting with a description of the traditional annual agricultural cycle. The structure of these verses corresponds with the typical properties of the Sui rhyming literature, already discussed in this author's previous article (2019). In the fragment presented below, the original verses in the left column are interlined with glosses abbreviated according to Leipzig Glossing Rules. All rhymes, combining intra- and inter-verse relations, are additionally marked with underlines. In order to preserve the original syllabic structure and rhymes, the English translation, placed in the right column, constitute a non-literal, poetic rendition of the original piece.

\begin{tabular}{|c|c|c|c|c|}
\hline $\begin{array}{l}\text { he }^{4} \\
\text { do }\end{array}$ & $\begin{array}{l}\text { ha: }{ }^{3} \\
\text { liquor }\end{array}$ & $\begin{array}{l}\text { qor }^{5} \\
\text { male ancestor }\end{array}$ & $\begin{array}{l}\text { tsje } e^{1} \\
\text { eat }\end{array}$ & Preparing offerings for an ancestor \\
\hline $\begin{array}{l}\text { ha }{ }^{1} \text { ii }^{3} \\
\text { EXCLAM }\end{array}$ & $\begin{array}{l}\text { pa: } u^{3} \\
\text { bless }\end{array}$ & & & Hani ${ }^{5}$ blessed! \\
\hline $\begin{array}{l}\text { ha }{ }^{1} \text { ii }^{3} \\
\text { EXCLAM }\end{array}$ & $\begin{array}{l}\underline{\mathrm{fu}}^{5} \\
\text { rich }\end{array}$ & & & Hani great! \\
\hline $\begin{array}{l}\underline{\text { nju }}^{4} \\
\text { this year }\end{array}$ & $\begin{array}{l}\mathrm{si}^{3} \\
\mathrm{be}\end{array}$ & $\begin{array}{l}\text { mbe }^{1} \\
\text { year }\end{array}$ & ${\underline{\operatorname{lda}: i^{1}}}^{\operatorname{good}}$ & Late year is bliss. \\
\hline$\frac{\text { na:i }^{2}}{\text { now }}$ & $\begin{array}{l}\mathrm{si}^{3} \\
\mathrm{be}\end{array}$ & $\begin{array}{l}\text { mbe }^{1} \\
\text { year }\end{array}$ & $\frac{\underline{\text { Pnjən }}^{3}}{\text { auspicious }}$ & This year is dear. \\
\hline$\frac{\operatorname{tən}^{3}}{\text { head }}$ & $\begin{array}{l}\mathrm{si}^{3} \\
\text { be }\end{array}$ & $\begin{array}{l}\text { qen }^{1} \\
\text { geng }\end{array}$ & $\frac{\underline{\mathrm{no}}^{2}}{w u}$ & Near is geng-wu \\
\hline $\begin{array}{l}\mathrm{p}^{\mathrm{h}} \mathrm{je}^{1} \\
\text { tail }\end{array}$ & $\begin{array}{l}\mathrm{si}^{3} \\
\mathrm{be}\end{array}$ & $\begin{array}{l}\underline{\text { tso }}^{2} \\
\text { auspicious }\end{array}$ & $\begin{array}{l}\text { nat }^{8} \\
\text { day }\end{array}$ & Now is clue day. \\
\hline $\begin{array}{l}\text { la: } k^{8} \\
\text { child }\end{array}$ & $\begin{array}{l}\text { pa: } i^{2} \\
\text { give birth to }\end{array}$ & $\begin{array}{l}\text { Pdai }{ }^{3} \\
\text { obtain }\end{array}$ & $\frac{\text { ta: } \eta^{3}}{\text { speak }}$ & Your heir comes plead. \\
\hline $\begin{array}{l}\text { la:k }{ }^{8} \\
\text { child }\end{array}$ & $\begin{array}{l}\text { ha: } \eta^{4} \\
\text { give birth to }\end{array}$ & $\begin{array}{l}\text { Pdai }^{3} \\
\text { obtain }\end{array}$ & 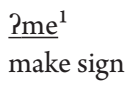 & Your seed comes pray. \\
\hline $\begin{array}{l}\operatorname{lən}^{2} \\
\text { behind }\end{array}$ & $\begin{array}{l}\mathrm{pu}^{3} \\
\text { also }\end{array}$ & $\frac{\text { mbe }^{1}}{\text { year }}$ & $\frac{\text { qa: } u^{5}}{\text { old }}$ & Old year's sway gone. \\
\hline$\frac{\underline{\mathrm{t}}^{\mathrm{h}} \underline{\mathrm{au}}^{5}}{\text { reach }}$ & $\begin{array}{l}\mathrm{pu}^{3} \\
\text { also }\end{array}$ & $\begin{array}{l}\text { mbe }^{1} \\
\text { year }\end{array}$ & $\frac{\operatorname{mai}^{5}}{\text { new }}$ & Don a new year. \\
\hline $\begin{array}{l}\text { mbe }^{1} \\
\text { year }\end{array}$ & $\frac{\operatorname{mai}^{5}}{\text { new }}$ & $\begin{array}{l}\text { Pdai }^{3} \\
\text { obtain }\end{array}$ & $\begin{array}{l}\underline{\text { na: }}^{6} \\
\text { exist }\end{array}$ & New year comes on. \\
\hline $\begin{array}{l}\text { mbe }^{1} \\
\text { year }\end{array}$ & $\frac{\text { qa: }^{5}}{\text { old }}$ & $\begin{array}{l}\text { Pdai }{ }^{3} \\
\text { obtain }\end{array}$ & $\frac{\mathrm{pa}^{1}}{\text { go }}$ & Old one leaves here. \\
\hline
\end{tabular}

\footnotetext{
${ }^{5}$ A function word marking the beginning or end of a paragraph.

6 In the Sui calendar, years, months and days are recorded with a Chinese-origin system of cardinals, comprising the set of ten Heavenly Stems (tiangan 天干) and twelve Earthly Branches (dizhi 地支). The Stems and Branches are combined to form cycles of sixty terms. Geng and wu are respectively the seventh of the Heavenly Stems and the seventh of the Earthly Branches.
} 


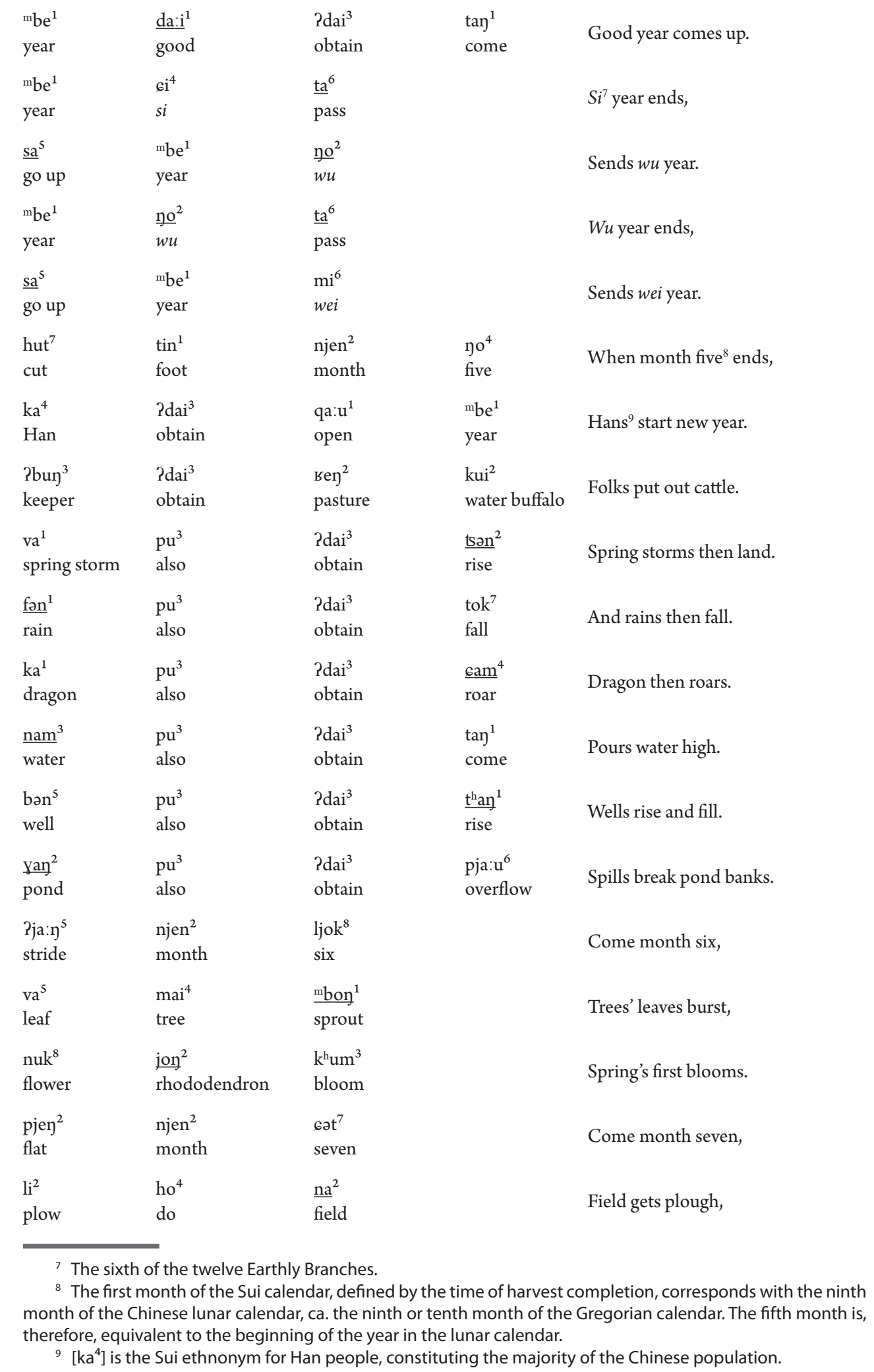




\begin{tabular}{|c|c|c|c|c|}
\hline $\begin{array}{l}\mathrm{pa}^{2} \\
\text { hoe }\end{array}$ & $\begin{array}{l}\text { ho } \\
\text { do }\end{array}$ & $\begin{array}{l}\frac{\text { Pya }}{5} \\
\text { field }\end{array}$ & & Now hoe ground. \\
\hline $\begin{array}{l}\underline{\text { sa }}^{5} \\
\text { go up }\end{array}$ & $\begin{array}{l}\text { njen }^{2} \\
\text { month }\end{array}$ & $\begin{array}{l}\text { pa:t }{ }^{7} \\
\text { eight }\end{array}$ & & Come month eight, \\
\hline $\begin{array}{l}\mathrm{au}^{4} \\
\text { grain }\end{array}$ & $\begin{array}{l}\text { pa:i }{ }^{1} \\
\text { go }\end{array}$ & $\frac{\mathrm{ta}^{3}}{\text { field }}$ & & One plants crops, \\
\hline $\begin{array}{l}\frac{\mathrm{dja}}{}^{3} \\
\text { rice seed }\end{array}$ & $\begin{array}{l}\text { pa:i }{ }^{1} \\
\text { go }\end{array}$ & $\begin{array}{l}\mathrm{ti}^{6} \\
\text { rice field }\end{array}$ & & Drops seeds through. \\
\hline $\begin{array}{l}\text { sa }^{5} \\
\text { go up }\end{array}$ & $\begin{array}{l}\text { njen }^{2} \\
\text { month }\end{array}$ & $\begin{array}{l}\mathrm{tu} \mathrm{u}^{3} \\
\text { nine }\end{array}$ & & Come month nine, \\
\hline $\begin{array}{l}\text { sui }^{3} \\
\text { Sui }\end{array}$ & $\begin{array}{l}\text { sot }^{7} \\
\text { say }\end{array}$ & $\begin{array}{l}\underline{\operatorname{lam}}^{1} \\
\text { insert seedlings }\end{array}$ & & Suis plan out, \\
\hline $\begin{array}{l}\operatorname{kam}^{1} \\
\text { Kam }\end{array}$ & $\begin{array}{l}\text { sot }^{7} \\
\text { say }\end{array}$ & $\begin{array}{l}\mathrm{ne}^{2} \\
\text { pluck }\end{array}$ & & Now Kams ${ }^{10}$ pluck. \\
\hline $\begin{array}{l}\text { sa }^{5} \\
\text { go up }\end{array}$ & $\begin{array}{l}\text { njen }^{2} \\
\text { month }\end{array}$ & $\begin{array}{l}\sup ^{8} \\
\text { ten }\end{array}$ & & Come month ten, \\
\hline $\begin{array}{l}\mathrm{au}^{4} \\
\text { rice }\end{array}$ & $\begin{array}{l}\text { tjen }^{6} \\
\text { become }\end{array}$ & $\begin{array}{l}\underline{\text { hon }}^{2} \\
\text { stem of a plant }\end{array}$ & & Rice pricks soil, \\
\hline$\frac{\text { qon }^{1}}{\text { work }}$ & $\begin{array}{l}\text { tjen }^{6} \\
\text { mount }\end{array}$ & $\begin{array}{l}\tan ^{3} \\
\text { end }\end{array}$ & & Toil is done. \\
\hline $\begin{array}{l}\mathrm{sa}^{5} \\
\text { go up }\end{array}$ & $\begin{array}{l}\text { njen }^{2} \\
\text { month }\end{array}$ & $\begin{array}{l}\text { sup }^{8} \text { pjat }^{7} \\
\text { eleven }\end{array}$ & & Come month eleven, \\
\hline $\begin{array}{l}\operatorname{tsin}^{5} \\
\text { stem }\end{array}$ & $\begin{array}{l}\mathrm{au}^{4} \\
\text { rice }\end{array}$ & $\begin{array}{l}\text { Pdai } \\
\text { obtain }\end{array}$ & $\begin{array}{l}\text { kwa: } \eta^{1} \\
\text { shining }\end{array}$ & Rice is well grown. \\
\hline $\begin{array}{l}\mathrm{sa}^{5} \\
\text { go up }\end{array}$ & $\begin{array}{l}\text { njen }^{2} \\
\text { month }\end{array}$ & $\begin{array}{l}\text { sup }^{8} \text { ni }^{6} \\
\text { twelve }\end{array}$ & & Now come month twelve, \\
\hline $\begin{array}{l}\text { mbja: }^{1} \\
\text { ear of grain }\end{array}$ & $\begin{array}{l}\mathrm{au}^{4} \\
\text { rice }\end{array}$ & $\begin{array}{l}\text { Pdai }^{3} \\
\text { obtain }\end{array}$ & $\begin{array}{l}\text { ma: } n^{3} \\
\text { yellow }\end{array}$ & Paddy rice ripens. \\
\hline $\begin{array}{l}\mathrm{sa}^{5} \\
\text { go up }\end{array}$ & $\begin{array}{l}\text { njen }^{2} \\
\text { month }\end{array}$ & $\begin{array}{l}\text { tsjen }{ }^{1} \\
\text { first }\end{array}$ & & Come month one, \\
\hline $\begin{array}{l}\mathrm{au}^{4} \\
\text { rice }\end{array}$ & $\begin{array}{l}\text { Pdai } \\
\text { obtain }\end{array}$ & $\begin{array}{l}\mathrm{ma}^{1} \\
\text { come }\end{array}$ & $\begin{array}{l}\text { ya: }{ }^{1} \\
\text { house }\end{array}$ & Grain fills store rooms. \\
\hline $\begin{array}{l}\text { pjen }^{2} \\
\text { flat }\end{array}$ & $\begin{array}{l}\text { njen }^{2} \\
\text { month }\end{array}$ & $\begin{array}{l}\text { ni }^{6} \\
\text { two }\end{array}$ & & Come month two, \\
\hline $\begin{array}{l}\text { va: } \eta^{1} \\
\text { straw }\end{array}$ & $\begin{array}{l}\text { Pdai } \\
\text { obtain }\end{array}$ & $\begin{array}{l}\mathrm{t}^{\mathrm{h}} \mathrm{au}^{5} \\
\text { reach }\end{array}$ & $\begin{array}{l}\text { ba: } n^{3} \\
\text { village }\end{array}$ & Straw piles in towns. \\
\hline $\begin{array}{l}\mathrm{sa}^{5} \\
\text { go up }\end{array}$ & $\begin{array}{l}\text { njen }^{2} \\
\text { month }\end{array}$ & $\begin{array}{l}\text { ha: } \mathrm{m}^{1} \\
\text { three }\end{array}$ & & Come month three, \\
\hline
\end{tabular}

10 The Kam people, also known as Dong 侗, are one of the ethnic minorities recognised by the People's Republic of China. Their main settlements are located in Guizhou Province (Guizhou Sheng 贵州省) and adjacent regions. 


\begin{tabular}{|c|c|c|c|c|}
\hline $\begin{array}{l}\text { in }^{5} \\
\text { swallow }\end{array}$ & $\begin{array}{l}\mathrm{t}^{\mathrm{h}} \mathrm{am}^{3} \\
\text { build }\end{array}$ & $\frac{\text { kun }^{1}}{\text { nest }}$ & & Nests fill trees, \\
\hline $\begin{array}{l}\text { yun } \\
\text { bee }\end{array}$ & $\begin{array}{l}\mathrm{th}^{\mathrm{h}} \mathrm{mm}^{3} \\
\text { build }\end{array}$ & $\begin{array}{l}\mathrm{fa}^{3} \\
\text { hive }\end{array}$ & & Bees build hives. \\
\hline $\begin{array}{l}\text { pjen }^{2} \\
\text { flat }\end{array}$ & $\begin{array}{l}\text { njen }^{2} \\
\text { month }\end{array}$ & $\begin{array}{l}6 i^{5} \\
\text { four }\end{array}$ & & Come month four, \\
\hline $\begin{array}{l}\text { sui }^{3} \\
\text { Sui }\end{array}$ & & $\begin{array}{l}\text { ljeu } \\
\text { ASPP }\end{array}$ & $\frac{\text { twa }}{}^{3}$ & New Year Sui got, \\
\hline$\frac{\mathrm{ka}^{4}}{\mathrm{Han}}$ & $\begin{array}{l}\mathrm{mi}^{4} \\
\mathrm{NEG}\end{array}$ & $\begin{array}{l}\mathrm{tsje}^{1} \\
\text { eat }\end{array}$ & $\begin{array}{l}\text { tsjey }^{1} \\
\text { first month }\end{array}$ & Not for Han yet. \\
\hline
\end{tabular}

\section{Bibliography}

Burkiewicz, Kamil 2015. The Script and Language of the Sui in Southern China: Description, Dictionary, Relations with Chinese Language and Culture. PhD dissertation. Poznań: Adam Mickiewicz University.

_ 2019. "Cosmogonic and anthropogenic myths in Sui oral literature". Litteraria Copernicana 2 (30): 183-195.

Wang Pinkui 1994. Zheng Qi Juan, Ren Chen Juan [The Volume of the First and Seventh Months, the Volume of the Ren and Chen]. Guiyang: Guizhou Minzu Chubanshe.

Wei Shuqi 2012. Shuizu "Jizu Jing" de Wenhua Jiedu - Yi Wei Chaoxian "Jizu Jing" wei Li [Cultural Interpretation of "The Volume of Ancestor Worship" - Basing on Wei Chaoxian's "Volume of Ancestor Worship”]. Master's thesis. Guiyang: Guizhou Minzu University.

11 [sje $^{1}$ twa $\left.^{3}\right]$, which literally means "to celebrate the end of a year", is one of the most popular and celebrated festivals among the Sui people. Performed after completion of the harvest season, it symbolically ends one cycle of farming works and starts another. 